\title{
Space-Time Coded MB-OFDM UWB System with Multi-channel Estimation for Wireless Personal Area Networks
}

\author{
Bon-Wook Koo, Myung-Sun Baek, Jee-Hoon Kim, and Hyoung-Kyu Song \\ uT Communication Research Institute, Sejong University, Seoul, Korea \\ bebop@sdc.sejong.ac.kr, sabman@sju.ac.kr, bid4u@sdc.sejong.ac.kr, \\ songhk@sejong.ac.kr
}

\begin{abstract}
In this paper, we apply multiple antennas to MB-OFDM UWB system for high performance. With an emphasis on a preamble design for multi-channel separation, we address the channel estimation in MB-OFDM system with multiple antennas. By properly designing each preamble for multiple antennas to be orthogonal in time domain, the channel estimation can be applied to the MB-OFDM proposal for IEEE 802.15.3a standard in the case of more than 2 transmit antennas. By using the multi-antenna scheme and proposed channel estimation technique, the reliability and performance of MB-OFDM system can be improved.
\end{abstract}

Keywords: MB-OFDM, CAZAC, multiple antennas, channel estimation.

\section{Introduction}

Ultra-wideband (UWB) technology is selected as a solution for the IEEE 802.15.3a standard [1] for low cost and high performance wireless entertainment network able to support streaming multimedia content and full motion video. In the standard of IEEE 802.15.3a, the data rate must be high enough (greater than 110 $\mathrm{Mb} / \mathrm{s}$ ) to satisfy a set of multimedia industry needs for wireless personal area networks (WPAN) communication. The standard also address the quality of service (QoS) capabilities required to support multimedia data types 1]. Therefore, higher rate and reliable transmission are required to satisfy the condition.

In this paper, as a solution for higher rate and reliable transmission, we apply MIMO architectures using space-time block code (STBC) to MB-OFDM UWB system. As an application of the MIMO architecture, a preamble structure for employing STBC with more than 2 transmit antennas is designed to be orthogonal in the time domain, and the channel estimation performance based on an investigated preamble structure is highlighted.

\section{Space-Time Coded MB-OFDM System}

In the WPAN system based on MB-OFDM, the whole available UWB spectrum between $3.1-10.6 \mathrm{GHz}$ is divided into 14 sub-bands with $528 \mathrm{MHz}$ bandwidth [2]. 
The transmission rate of the MB-OFDM is between $53.3-480 \mathrm{Mbps}$. In each subband, a normal OFDM modulated signal with 128 subcarriers is used. The main difference between the MB-OFDM system and other narrowband OFDM systems is the way that different sub-bands are used in the transmission with several hopping patterns.

As mentioned previously, for high performance of the system, we apply multiple antennas (2 and 4 transmit antennas and 1 receive antenna) to MB-OFDM UWB system. In this paper, we use the STBC proposed by Alamouti [3].

\section{Preamble Design for Multiple Antennas of MB-OFDM}

In the STBC MB-OFDM system, the channel estimation is carried out for every transmit antenna of the all sub-band. For the channel estimation of each antenna, we design preambles using the Zadoff-Chu sequence, one of the constantamplitude zero-autocorrelation (CAZAC) sequence, 4. The sequence $C_{k}$ is as follows :

$$
C_{k}=\exp \left[j \frac{M \pi k^{2}}{N}\right]
$$

where $N$ is a length of preamble, and $k=[0,1, \ldots, N-1] . M$ is an integer relatively prime to $N$, and we consider the case of $M$ is 1 .

$C_{k}$ has the property of a periodic autocorrelation function that is zero everywhere except at a single maximum per period. In the MB-OFDM system, The length of the channel estimation sequence is 128 except zero-padded suffix and guard interval. By using $C_{k}$ of 64 -symbol length $(N=64)$, we design the extended 128-length Zadoff-Chu sequence with zero-padding. The extended ZadoffChu sequence is as follows:

$$
S_{8 m+n+1}=\left\{\begin{array}{cl}
C_{4 m+n} & \text { for } m: \text { even including zero } \\
0 & \text { for } m: \text { odd }
\end{array}\right.
$$

where $m \in\{0,1,2, \ldots, 15\}, n \in\{0,1,2, \ldots, 7\}$. Fig. 1 shows the structure of preambles. In order to apply 4 transmit antennas, 4 preambles can be designed by cyclic shift. For 2 transmit antennas, P1 and P3 preambles are used, and for 4 transmit antennas, all preambles are used.

From [5], we deduce generalized relation to determine the number of distinguishable paths $D$ as follows:

$$
1 \leq D \leq \frac{L}{N_{t}}
$$

where $L$ indicates the symbol-length of sequence, and $N_{t}$ is the number of transmit antenna.

The orthogonality of preambles is broken and the preamble is not suitable for MB-OFDM specification, when the system just uses 64-symbol length sequence. However, extended sequences will keep the property of the orthogonality at channel model (CM) 1 and 2 when the system uses 2 and 4 transmit antennas. It is noted that $D$ is 64 for $N_{t}=2$ and is 32 for $N_{t}=4$ from (3). Using the 


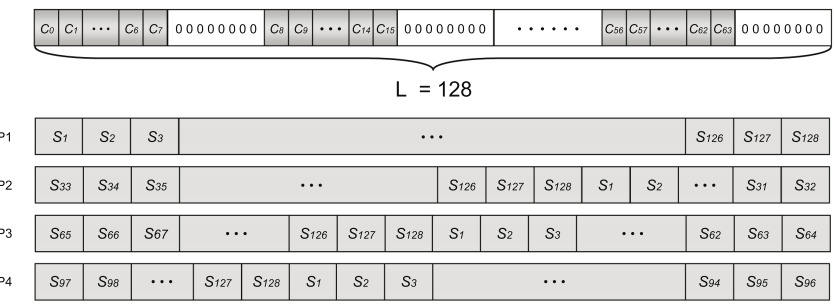

Fig. 1. The structure of each 128 length preamble for 4 transmit antennas system

orthogonality, the receiver can execute the channel estimation and separate channel impulse response (CIR) of each transmit antenna.

\section{Performance Evaluation and Discussion}

The performance of the proposed preamble is evaluated in terms of MSE and BER in this section. Fig. 2 (a) shows the MSE performance of 2 and 4 transmit antennas at CM 1-4 6]. In the case of 2 and 4 transmit antennas of CM 1 and 2 transmit antennas of CM2, the system can keep the orthogonality of preambles. However, in the other cases, MSE performances are very poor because the orthogonality of preamble is broken because of the reason that mentioned through (3) in section 3 .

Fig. 2 (b) shows the effect of the number of transmit antennas on the BER performance. Simulations are executed in conditions which are 1, 2 and 4 trans-

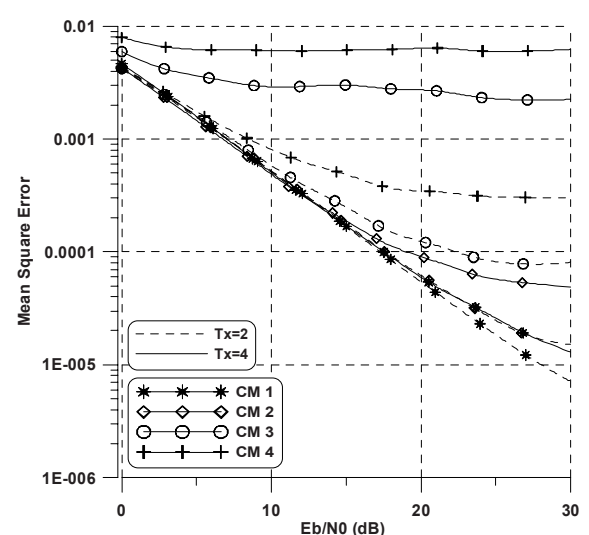

(a)

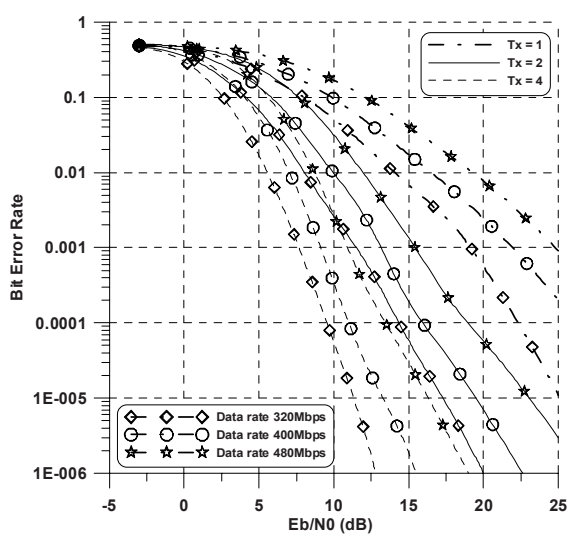

(b)

Fig. 2. (a) The MSE performance of proposed preambles applied to 2 and 4 transmit antennas with MMSE estimator. (b) The BER performance 1, 2 and 4 transmit antennas. 
mit antennas at data rate 320,400 and 480Mbps. Simulation results show that multi-antenna systems have the better performance than single antenna system and also as the number of antennas increases, the system has the better BER performance.

\section{Conclusions}

In this paper, we apply space-time architecture to MB-OFDM system based on WPAN for high capacity transmission and propose the new preamble structure for channel estimation which is required in MIMO architecture. Through the MSE performance, simulation results have shown that the proposed sequence can be adopted to multi-antenna MB-OFDM system. The BER performance shows that the reliability of STBC MB-OFDM system is improved efficiently by increasing the number of antennas. As the new preamble is applied, it has been shown that the MB-OFDM system with multi-antenna can achieve the high transmission capacity.

\section{Acknowledgement}

This work is financially supported by the Ministry of Education and Human Re-sources Development(MOE), the Ministry of Commerce, Industry and En$\operatorname{ergy}(\mathrm{MOCIE})$ and the Ministry of Labor(MOLAB) through the fostering project of the Lab of Excellency and is supported by MIC Frontier R\&D Program in KOREA.

\section{References}

1. "IEEE 802.15 WPAN high rate alternative PHY Task Group 3a(TG3a)[Online]," Available: http://www.ieee802.org/15/pub/TG3a.html

2. MultiBand OFDM Alliance(MBOA) Special Interest Group(SIG), WiMedia Alliance, Inc.(WiMedia) "MultiBand OFDM Physical Layer Specification,"Release 1.1, July 2005.

3. Siavash M. Alamouti, "A Simple Transmit Diversity Technique for Wireless Communications," IEEE Journal on Selected Areas in Communications, vol. 16, no. 8, pp. 1451-1458, October 1998.

4. David C. Chu, "Polyphase Codes With Good Periodic Correlation Properties," IEEE Transactions on information theory, vol. 18, no. 4, pp. 531-532, July 1972.

5. Dong-Jun Cho, Young-Hwan You, and Hyoung-Kyu Song, "Channel Estimation with Transmitter Diversity for High Rate WPAN Systems," IEICE Trans. Commun., vol. E87-B, no. 11, Nov. 2004.

6. IEEE P802.15-02/490rl-SG2a, "Channel Modeling Sub-committee Report Final," February 2003. 